\title{
Declining diabetes education: the views of young people with type 1 diabetes, parents and health care professionals
}

\section{V.Coates ${ }^{1}$, G.Horigan², M.Davies ${ }^{3}$, F.Findlay White ${ }^{4}$, M.Carey ${ }^{5}$}

${ }^{1}$ Ulster University, School of Nursing, Coleraine, Northern Ireland and Western Health and Social Care Trust, ${ }^{2}$ Northern Ireland Clinical Research Services Ltd, Ulster University, Londonderry, Northern Ireland, ${ }^{3}$ Belfast Health and Social Care Trust, Clinical Psychology, Belfast, Northern Ireland, ${ }^{4}$ Diabetes UK, Care Advisor, Belfast, Northern Ireland, ${ }^{5}$ Director: Structured Education Research Portfolio, Honorary Associate Professor, Diabetes Research Centre, University of Leicester.

\section{Background}

"The UK has the fourth highest number of children and young people in Europe with Type 1 diabetes, but is one of the worst performing countries in terms of blood glucose control" [1].

And yet - despite evidence of the effectiveness of education and self-management support structured diabetes education (SDE) is declined by a significant proportion of those invited to attend [2]. The National (UK) Paediatric Diabetes Audit 2013/14 reported less than $40 \%$ of children and young people were recorded as having received some form of such education [3].

\section{Aim}

To identify factors that influence lack of engagement of young people with type 1 diabetes (aged 13-21 years) with structured diabetes education to inform and broaden the reach of future SDE from a sound evidence base.

\section{Method}

A two-phase study was completed.
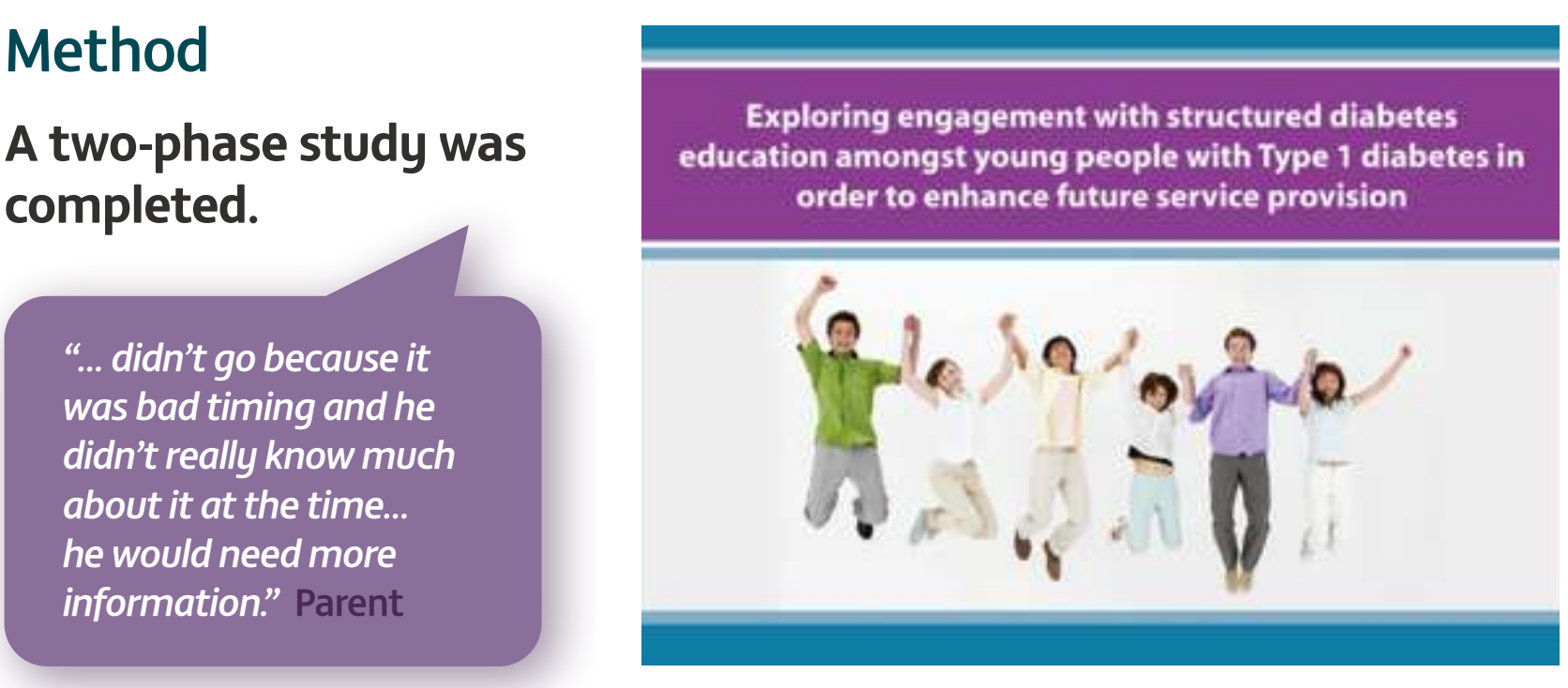

Phase 1: Cross sectional survey from 11 health trusts across Northern Ireland (NI) and England, (United Kingdom) $(n=227)$. A questionnaire exploring non-attendance, health and diabetes specific variables was completed and $\mathrm{HbA} 1 \mathrm{c}$ results collected [4].

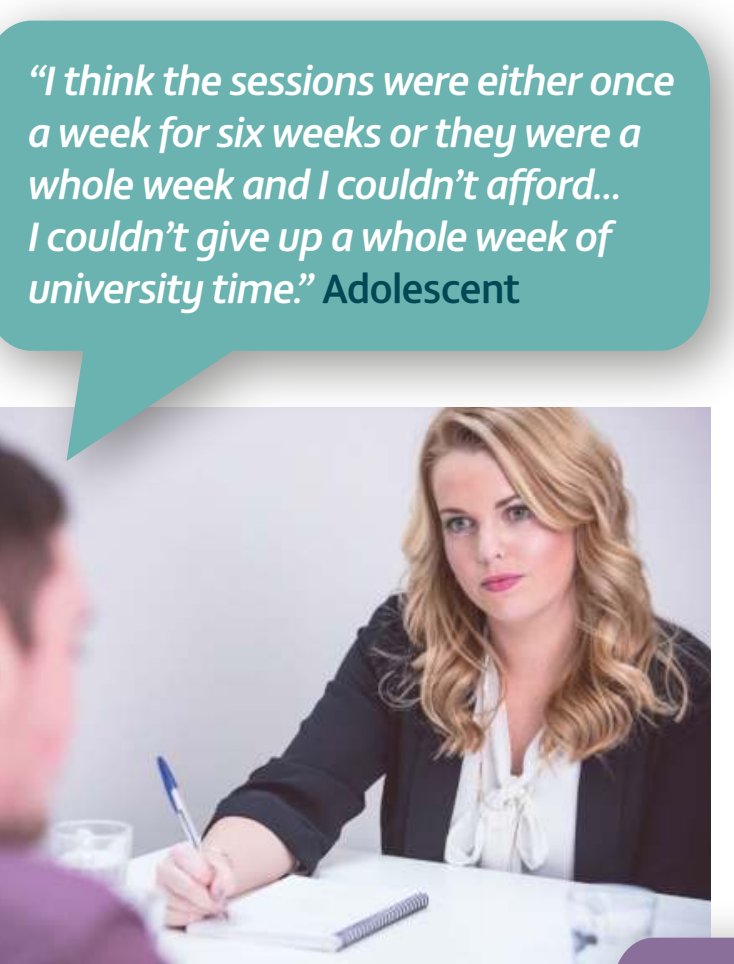

Phase 2: Interviews were conducted by two researchers (GH and $\mathrm{MC}$ ) with adolescents $(n=20)$, parents $(n=17)$ and health care professionals $(n=16)$ to explore the reasons for non-attendance at SDE in depth. Each interview lasted 30-40 minutes and focus groups lasted 45-60 minutes. They were digitally recorded, transcribed and a thematic analysis was undertaken (by $\mathrm{GH}$, $M D, V C)$. Each emerging theme was compared between the research team until consensus was reached.

\section{Results}

The mean $\mathrm{HbA} 1 \mathrm{c}$ of the entire sample was $76.7( \pm$ SD 20.0) $\mathrm{mmol} / \mathrm{mol}(9.2 \%)$.

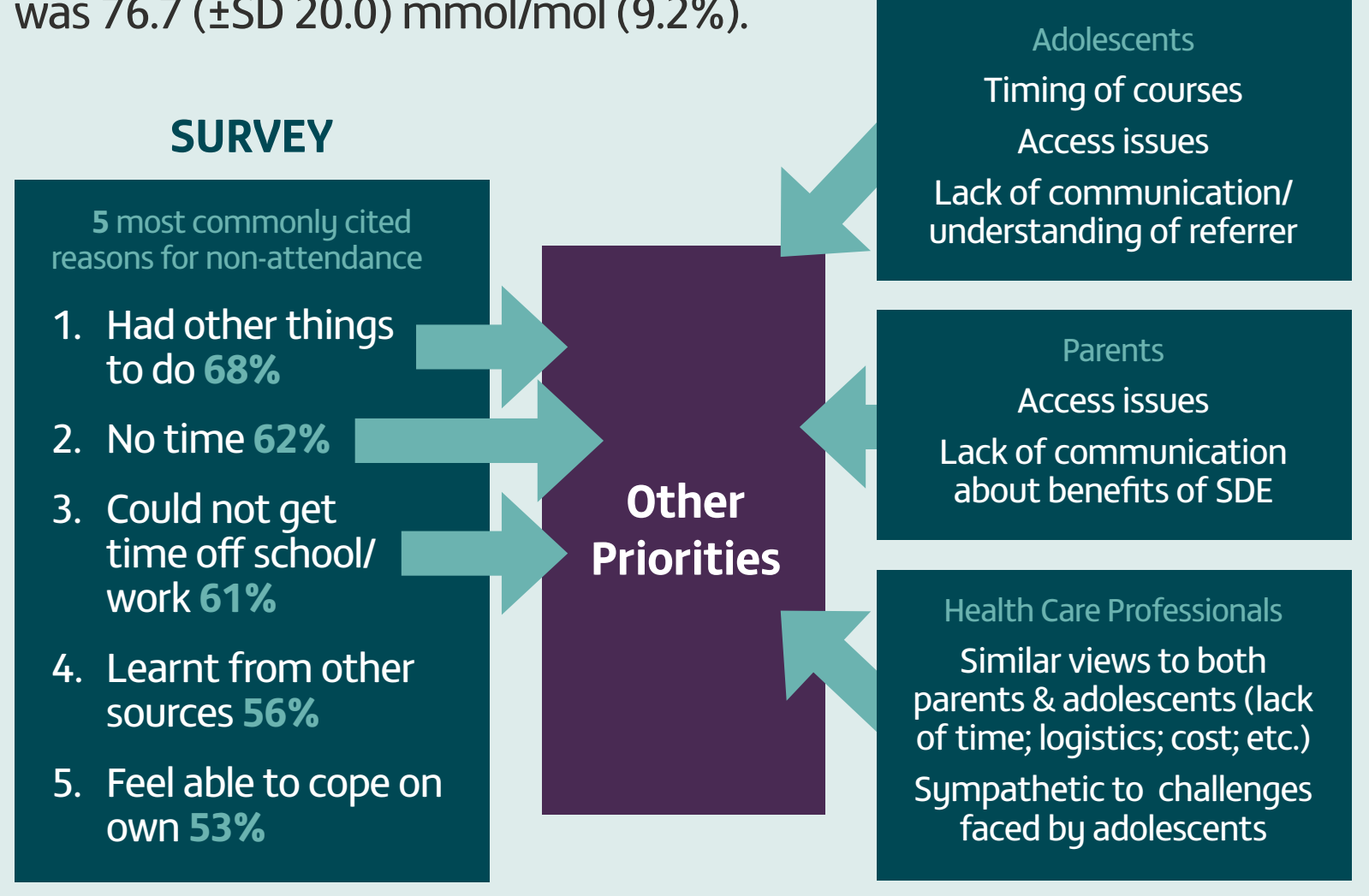

\section{Conclusion}

This study, spanning both quantitative and qualitative approaches provided the opportunity to gather data that can be genenralised (the survey) and also to probe and gain direct information from these three cohorts (the interviews). From the survey it appears as though other issues take priority. However, the qualitative results showed that SDE may be declined due to timing and location of courses, some require complicated travel arrangements. Discussion with those adolescents, their parents and HCPs revealed that online modes of education should be explored alongside consideration being given to tailored approaches to individuals with specific needs. Further investigation is required into preferred, effective modes of delivery of SDE for those with type 1 diabetes without losing the quality of structured programmes. Bearing in mind that many of the young adults involved in this study had sub-optimal glycaemic control, solutions to non-attendance are urgently required.

didn't go because it was bad timing and he didn't really know much about it at the time... he would need more information." Parent

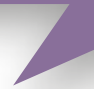

\section{References}

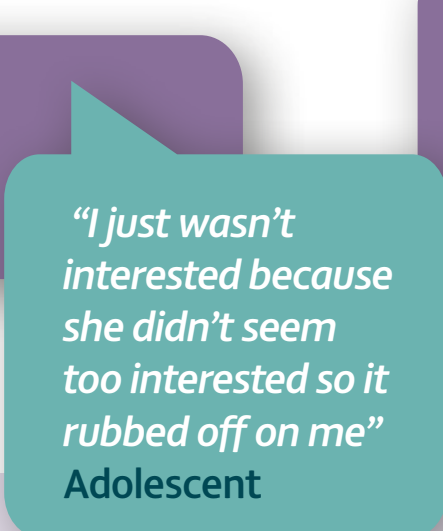

"Work is awkward to get off as I have to be fully flexible" Parent

1. Diabetes UK (2015) Position Statement: High quality care for children and young people Diabetes UK website.

2. Horigan et al Reasons why patients referred to diabetes education programmes choose not to attend: a systematic review. Diabetic Medicine. (2017) 34, 1; 14-26.

3. http://www.rcpch.ac.uk/sites/default/files/page/NPDA\%20Report\%202014-15\%20v5.2\%20 sent\%20to\%20HQIP\%2025.05.2016.pdf

4. Coates, et al. Educational and psychological aspects why young people with Type 1 diabetes decline structured education with a view to overcoming barriers. Diabetic Medicine. (2017) 34 (8), 1092-1099.. DOI: 10.1111/dme.13368 